\title{
1 Genome-wide analysis of mobile element 2 insertions in human genomes
}

3 Running title: Mobile element insertion map of 5,675 genomes

4 Yiwei $\mathrm{Niu}^{1,2,5}$, Xueyi Teng ${ }^{1,3,5}$, Yirong Shi ${ }^{1,3}$, Yanyan $\mathrm{Li}^{1,2}$, Yiheng Tang ${ }^{1,3}$, Peng Zhang ${ }^{1}$, Huaxia

$5 \quad$ Luo $^{1}$, Quan Kang ${ }^{1}$, The Han100K Initiative ${ }^{\S}$, Tao $\mathrm{Xu}^{2,4^{*}}$, Shunmin $\mathrm{He}^{1,3,6^{*}}$

6

71 Key Laboratory of RNA Biology, Center for Big Data Research in Health, Institute of Biophysics,

8 Chinese Academy of Sciences, Beijing 100101, China.

92 College of Life Sciences, University of Chinese Academy of Sciences, Beijing 100049, China.

3 University of Chinese Academy of Sciences, Beijing 100049, China.

114 National Laboratory of Biomacromolecules, CAS Center for Excellence in Biomacromolecules,

5 These authors contributed equally to this work.

Keywords: mobile element insertion, MEI, transposable element, whole genome sequencing, 


\section{Abstract}

Mobile element insertions (MEIs) are a major class of structural variants (SVs) and have been linked to many human genetic disorders, including hemophilia, neurofibromatosis, and various cancers. However, human MEI resources from large-scale genome sequencing are still lacking compared to those for SNPs and SVs. Here, we report a comprehensive map of 36,699 nonreference MEIs constructed from 5,675 genomes, comprising 2,998 Chinese samples ( 26.2X, NyuWa) and 2,677 samples from the 1000 Genomes Project ( 7.4X, 1KGP). We discovered that LINE-1 insertions were highly enriched at centromere regions, implying the role of chromosome context in retroelement insertion. After functional annotation, we estimated that MEIs are responsible for about $9.3 \%$ of all protein-truncating events per genome. Finally, we built a companion database named HMEID for public use. This resource represents the latest and largest genomewide study on MEIs and will have broad utility for exploration of human MEI findings.

\section{Introduction}

Transposable elements (TEs), also known as transposons or mobile elements, comprise a significant portion in mammalian genomes (Smit 1999; Deininger et al. 2003; Cordaux and Batzer 2009), approximately half of the human genome (Lander et al. 2001). Most TEs are transposition incompetent due to accumulated interior mutations and truncation or various host repression mechanisms (Goodier 2016). In humans, Alu, long interspersed nuclear element 1 (L1), SINE-VNTR-Alu (SVA), and HERV-K (also known as HML-2) are four families of TEs which are still active and capable of creating new insertions (Mills et al. 2007; Huang et al. 
2012), termed mobile element insertions (MEIs). The transposition events have the potential to disrupt normal gene function and alter transcript expression or splicing at the sites of integration, contributing to disease (Payer and Burns 2019). For example, over 120 TE-mediated insertions have been associated with various human genetic diseases, including hemophilia, Dent disease, neurofibromatosis and various cancers (Hancks and Kazazian 2016). Apart from the impact through insertion events, intrinsic sequence properties of TEs endow some MEIs with functional effects on the host (Payer and Burns 2019), making MEIs differ qualitatively from typical forms of SVs like copy number variants (CNVs). Another important question related to MEIs is the integration site preference, which are usually non-random and influenced by various factors such as DNA sequences and chromatin context (Sultana et al. 2017).

However, despite these important functions, integrated resources for polymorphic TEs in human genomes is still lacking (Goerner-Potvin and Bourque 2018), which could offer a large pool of MEIs to explore TE diversity and serve as bedrock for phenotype-variant association studies. And MEIs are not routinely analyzed in most population-scale whole-genome sequencing (WGS) projects (The 1000 Genomes Project Consortium 2015; Wu et al. 2019, 2019; Cao et al. 2020). To date, the largest and most recent population study of MEIs using WGS remains the one conducted by the 1KGP, which included 2,504 genomes across 26 human populations (Sudmant et al. 2015; Gardner et al. 2017). However, the sequencing depth of the 1KGP is low, which may limit the MEI detection sensitivity and accuracy (Rishishwar et al. 2016). In addition, current MEI genetic resources are mainly from European ancestry cohorts, and the lack of Chinese cohort genomic study on MEIs is a critical part of the missing diversity. In this study, we employed WGS of 5,675 members from newly sequenced Chinese 
samples and the 1KGP to construct a resource for non-reference MEIs. Although the 1KGP dataset has already been investigated for MEIs (Sudmant et al. 2015; Gardner et al. 2017), we included it here to increase population diversity and build a comprehensive MEI map. The NyuWa dataset has been used to study spectrum of small variant and build reference panel (Zhang et al. 2020), and the MEIs were not explored yet. Combining two cohorts enabled us to systematically analyze the genomic distribution, mutational patterns, and functional impacts of MEIs. From these analyses, we found that L1 MEIs were highly enriched in centromere regions, and we determined that MEIs represent about $9.3 \%$ of all protein-truncating events per individual, emphasizing the importance of detecting MEI routinely in WGS studies. We have built a companion database named HMEID (available at http://bigdata.ibp.ac.cn/HMEID/) for polymorphic MEIs, which could be explored for new insights into MEI biology.

\section{Results}

\section{A Comprehensive Map of Non-reference Human MEIs}

To generate a comprehensive map of MEIs from human genomes, we jointly analyzed two WGS datasets using MELT (Gardner et al. 2017), the low-coverage 1KGP dataset consisting of 2,677 individuals sequenced to $\sim 7.4 \mathrm{X}$ coverage (Sudmant et al. 2015) and the high-coverage NyuWa dataset including 2,998 Chinese samples sequenced to $\sim 26.2 \mathrm{X}$ coverage (Table S1) (Zhang et al. 2020). After site quality filtering, a total of 36,699 non-reference MEIs were kept, including 26,553 Alus, 7,353 L1s, 2,667 SVAs and 126 HERV-Ks (Table 1). Most Alu and L1 MEIs were well-supported by split reads (Fig. S1A) and target site duplications (TSDs) (Fig. S1B). Using Hardy-Weinberg equilibrium (HWE) metrics as a rough proxy of genotyping 
accuracy, we found that about $87 \%$ autosomal MEI sites did not violate the HWE, and when restricted to the NyuWa dataset, almost all MEIs (97\%) on autosomes had high genotyping accuracy (Fig. S2).

Table 1. MEI discovery in this study.

\begin{tabular}{llllll}
\hline & & \multicolumn{2}{c}{ Mean sites per donor } & \multicolumn{2}{c}{ Standard deviation } \\
\cline { 3 - 6 } & Total sites & NyuWa & 1 KGP & NyuWa & 1 KGP \\
\hline Alu & 26,553 & 1,035 & 884 & 25.3 & 153 \\
LINE-1 & 7,353 & 145 & 119 & 8.35 & 19.3 \\
SVA & 2,667 & 44.4 & 28.8 & 4.83 & 9.9 \\
HERVK & 126 & 11 & 8.23 & 1.86 & 2.12 \\
Total & 36,699 & 1,236 & 1,040 & 30 & 178 \\
\hline
\end{tabular}

On average, we detected 1,236 MEIs with each genome in the NyuWa dataset and 1,040 MEIs in the 1KGP dataset (Table 1), which were expected as increased sequencing depth provides more power for MEI detection (Fig. S1C). The smaller correlation between MEI number and sequencing coverage in the NyuWa dataset than that of the 1KGP dataset reflected that MEI detection sensitivity was close to saturation in $~ 30 \mathrm{X}$ genomic coverage, consistent with the previous evaluation by the authors of MELT (Gardner et al. 2017). The distribution of MEI numbers per individual, MEI allele frequencies and length estimates largely fit the findings of previous studies (Fig. 1) (Gardner et al. 2017, 2019). About 70.7\% MEIs are very rare (allele frequency $<0.1 \%$ ), with over $30 \%$ singletons of all four MEI types (Fig. 1C; Fig. S1D). Since a large proportion of MEIs were individual-specific, we next sought to evaluate MEI discovery by increasing sample size. Through randomly down-sampling to different sizes with 100 - 
sample intervals, we estimated the total MEI variants and the increase of variants at different sample sizes (Fig. S1E-I). As expected, we found that the number of all four MEI types continued to rise with the increasing sample size, but the growth rate decreased. When looking at the subfamilies of MEIs, we found that the distributions of active Alu and L1 MEIs were in line with previous observations in humans (Gardner et al. 2017; Bennett et al. 2008; Stewart et al. 2011; Hormozdiari et al. 2013), e.g. AluYa5 and AluYb8 were found to be the most abundant two Alu subfamilies (Fig. S3), indicating their high retrotransposition activity in modern humans.
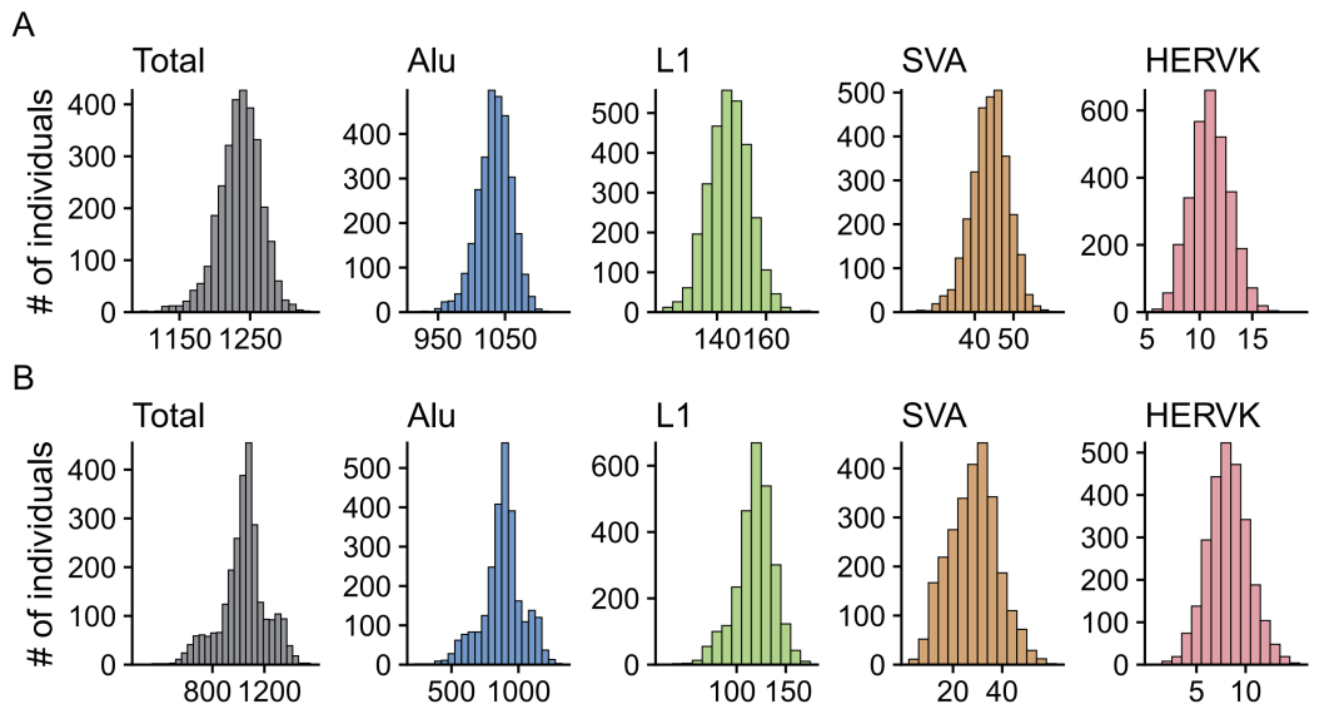

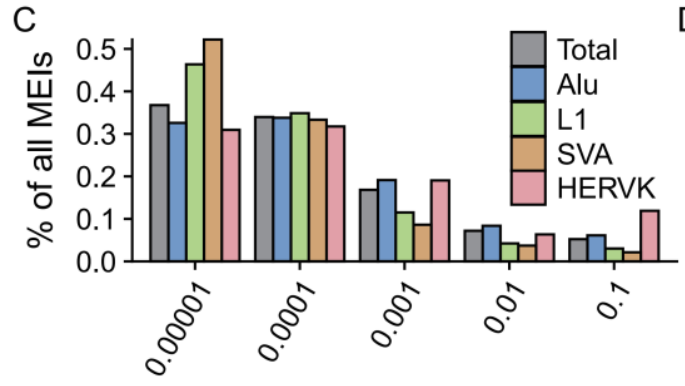

Allele frequency bins

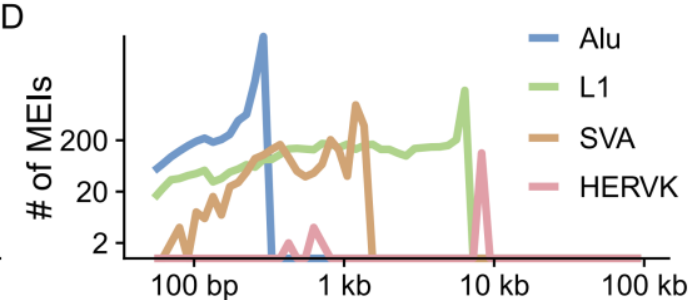

Insert length

Fig. 1. The MEI call set. (A) Histograms of the number of MEIs identified per genome in the NyuWa dataset. (B) Histograms of the number of MEIs identified per genome in the 1KGP dataset. (C) Distribution of allele frequency of MEIs of four types: Alu, L1, SVA, and HERVK. "Total" combined the four types of MEIs. (D) Distribution of insert size estimated by MELT. 
MEI calls detected by previous study were repeatedly identified in this study, and the allele coefficient $=0.95)$. Nonetheless, we noticed that many MEIs identified by Gardner et al.

(Gardner et al. 2017) were missed in our call set. We conjectured that this may be due to generated etc. To test this, we performed three runs using three sample sets: 1) 100 samples

MEI detection sensitivity as well as accuracy, with finer resolution of MEI break points.

Collectively, our MEI call set represents a high-quality map of non-reference MEIs for humans.

\section{Enrichment of Non-reference $L 1$ insertions in Centromeres}

132 It has been long noted that L1s occur preferentially in AT-rich regions but Alus show the 
S5A). In addition, the GC content of flanking DNA for Alus and L1s were lower than background, while SVAs and HERV-Ks prefer DNA sequences with much higher GC content.

We next compared the GC composition of rare MEIs (allele frequency $<1 \%$ ) and common MEIs (allele frequency $>=1 \%$ ) due to the reported bias shift in GC bias for older and younger short interspersed nuclear elements (SINEs) (Smit 1999; Hormozdiari et al. 2013; Medstrand et al. 2002; Waterson et al. 2005). Significant difference was only observed for HERV-K: rare HERV-K insertions occurred in much higher density at GC-rich regions (Fig. S5B). We did not observe marked bias for Alus and SVAs, likely because most insertions we identified were already fixed in population.

We next sought to investigate the distribution of MEIs throughout the genome, like previously Collins et al. had done for common SVs (Collins et al. 2020). Interestingly, L1s were predominantly enriched at centromeric regions, whereas SVAs and HERV-Ks were enriched at telomeres (Fig. 2 A and B; Fig. S6). For comparison, similar analysis was applied to TEs in the reference genome, but no such patterns for L1s were found (Fig. S7B). Even in the latest telomere-to-telomere assembly of the human X chromosome, only a single L1 insertion was detected at the centromere region (Miga et al. 2020). When restricted to singleton L1 MEIs, we could still detect the enrichment in centromeres (Fig. 2C). Importantly, this finding was wellsupported by non-reference L1s from euL1db (Fig. 2D) (Mir et al. 2015), which curated human polymorphic L1s from 32 different studies. Considering the reduced detection power of shortread WGS in repetitive regions, the enrichment of L1 insertions at centromeric regions could be still underestimated. The enrichment of non-reference L1 insertions at centromeric DNA could be partly attributed to lower GC content, as centromeres contain massive AT-rich alpha 

centromere regions are intriguing and further studies are needed in the future.

A

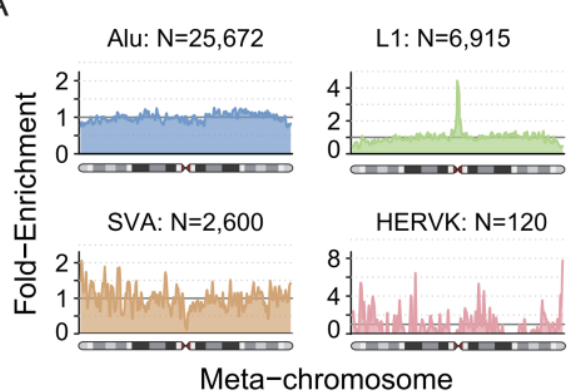

C

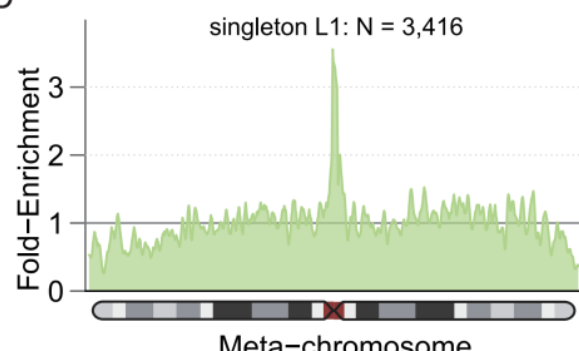

B
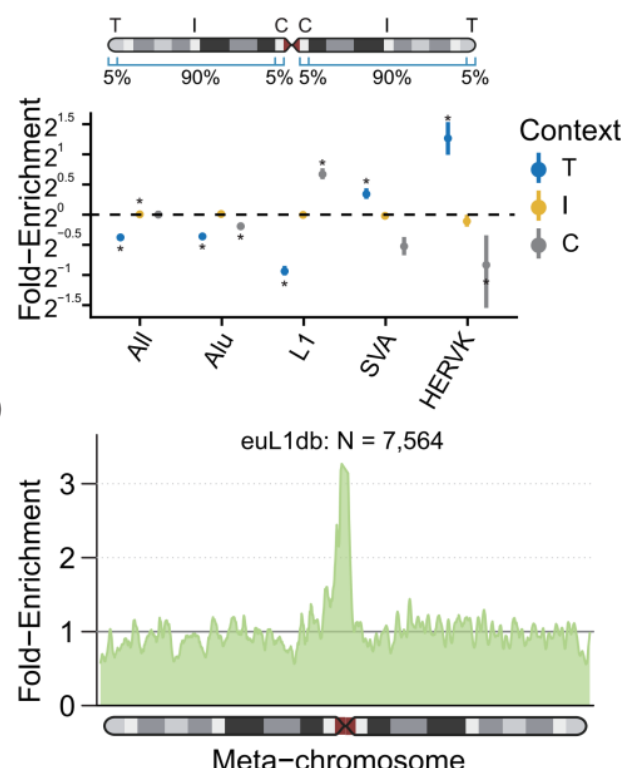

160

161

Fig. 2. Chromosome-level Distribution of MEI Density. (A) Smoothed enrichment of different types of MEIs ascertained in this study. The values were calculated per $100 \mathrm{~kb}$ window across the average of all autosomes and normalized by the length of chromosome arms (as "meta-chromosome"). Enrichment of MEIs by class and chromosomal context. The dots are the mean values and point ranges represent $95 \%$ confidence intervals (CIs). P-values were computed using a two-sided t-test and adjusted using the Bonferroni method. *, $\mathrm{p} \leq 0.05$. C, centromeric; I, interstitial; T, telomeric. The way to compute the chromosomal enrichment and to represent data was from the gnomAD SV paper (Collins et al. 2020). (C) Smoothed enrichment of singleton L1s (L1 MEIs found in single genome) ascertained in this study. (D) Smoothed enrichment of non-reference L1s from euL1db database (Mir et al. 2015). 


\section{Strong Correlations between MEI Diversity and SNP Heterozygosity}

171 Since mutations are ultimate sources of genetic innovation and significant causes of human

Waterson's estimator (Watterson 1975) of $\Theta$ to estimate the mutation rate of each MEI type and et al. 2019). 
A

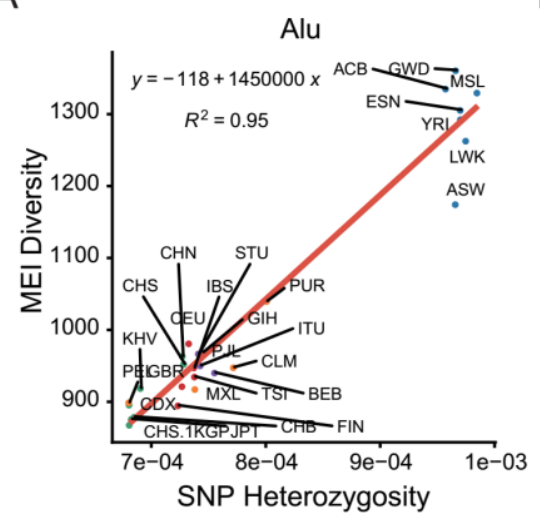

C

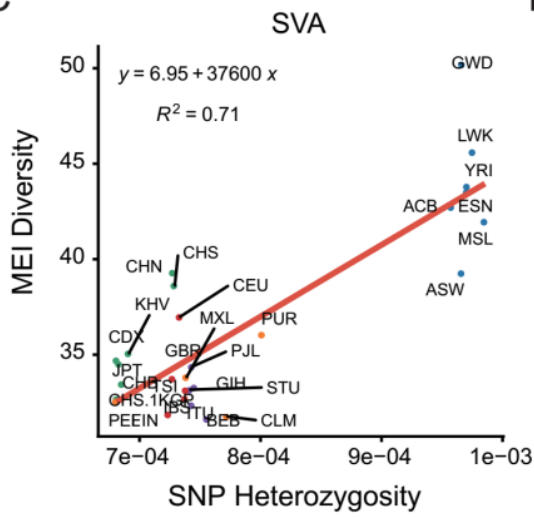

B

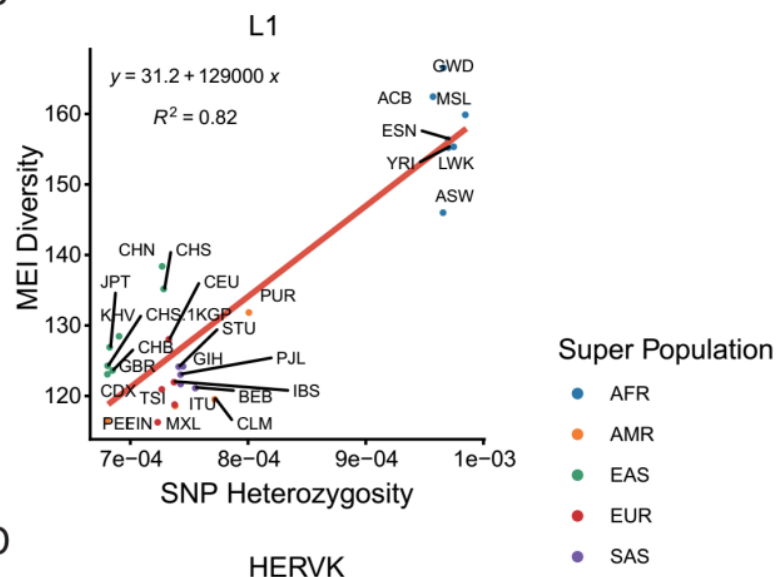

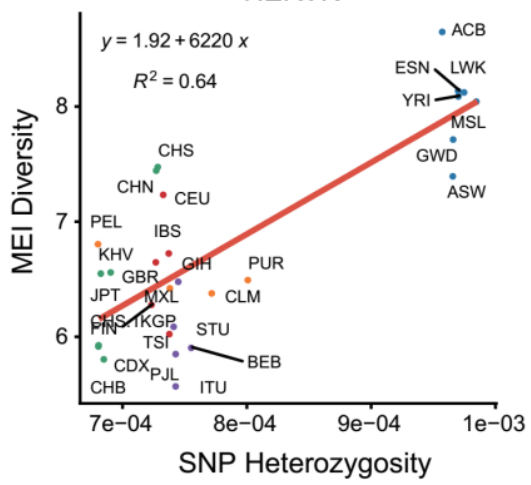

191 Fig. 3. Correlation between SNP heterozygosity and MEI diversity. SNP heterozygosities and diversity of (A) Alu MEIs, (B) L1 MEIs, (C) SVA MEIs and (D) HERV-K MEIs were compared in different populations. SNP heterozygosity was computed as the ratio of heterozygous SNPs across the individual's genome and MEI diversity was computed as the average allele difference in each population. super population. 
of-function (pLoF) by altering open-reading frames. To assess the functional impacts of MEIs, we annotated the MEI calls using Variant Effect Predictor (VEP) and BEDtools (see Methods). The vast majority (82.7\%) of detected MEIs was in intergenic and intronic regions, while only $\sim 2.7 \%$ MEIs impacted the coding sequences (CDS) (Fig. 4A). Varying enrichment levels on different genomic features were observed for different MEI types (Fig. 4B). For example, L1, SVA and HERV-K MEIs were significantly depleted in CDS and non-coding gene exons; L1 MEIs were enriched in coding introns and gene flanking regions; SVA and HERV-K sites were enriched in intergenic and non-coding introns. Focusing on protein-truncating variants (PTVs), each genome contained a mean of 24.8 MEIs (12.6 Alu, 7.4 L1, 1.3 SVA and 2.4 HERV-K) directly disrupting CDS, including 1.1 rare pLoF MEIs (allele frequency $<1 \%$ ) (Fig. 4C; Table S4). By comparison, Karczewski et al. estimated 98.9 pLoF short variants (SNVs and InDels) per genome (Karczewski et al. 2020), and Collins et al. observed 144.3 pLOF SVs per genome (Collins et al. 2020). We thus estimated that MEIs account for about 9.3\% (24.8/268) of all PTVs, among small variants and large SVs in each human genome.

Examining the degree to which evolutionary forces acting on coding MEI loci is important to understand the relationships between MEI variation and coding genes. Here we used three different metrics to investigate selective constraints: 1) the proportion of singleton variants (variants observed in only one individual), an established proxy for selection strengths (Lek et al. 2016); 2) the proportion of MEIs in genes with high probability of loss-of-function intolerance (pLI) (Lek et al. 2016); 3) the loss-of-function observed/expected upper bound fraction (LOEUF) of MEI-containing coding genes, where higher LOEUF scores suggest a relatively higher tolerance to inactivation for a given gene (Karczewski et al. 2020). HERV-K 
224 MEI was not included in this analysis due to the relatively small number found in coding genes.

225 Higher singleton proportions for Alu and L1 MEIs were found in CDS than that of introns (Fig.

$\left.2264 \mathrm{D} ; \chi^{2} \mathrm{p}<0.05\right)$, while we did not find a statistically significant bias for SVA MEIs, though

227 there were 166 and 949 SVA insertions found in CDS and coding introns, respectively. Likewise,

228 lower proportions of $A l u / \mathrm{L} 1$ MEIs detected in genes with high pLI score $(>0.9)$ were found in

229 CDS than that of intronic regions (Fig. 4E; $\chi^{2} p<0.05$ ). Observations from the perspective of

230 enclosing genes fit these results: higher LOEUF score were found for genes with $A l u /$ L1 MEIs

231 (Fig. 4F, Wilcoxon $\mathrm{p}<0.05$ ). Our results sustained and expanded previous findings on human

232 exome data (Gardner et al. 2019), in which Gardner et al. reported that exonic MEIs were under purifying selection. 
A

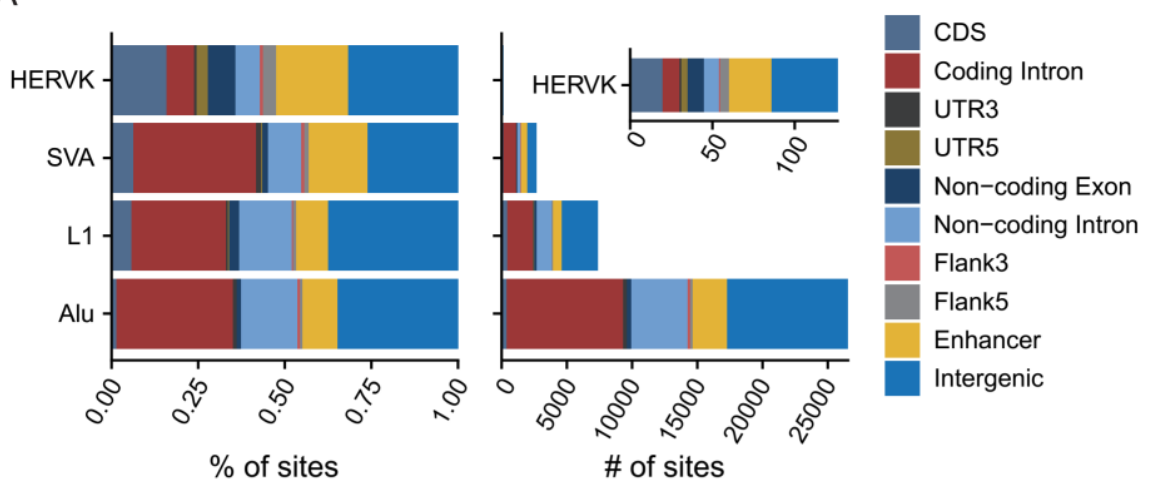

B

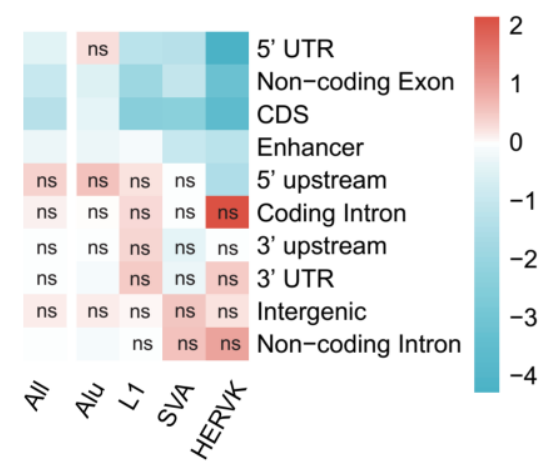

D

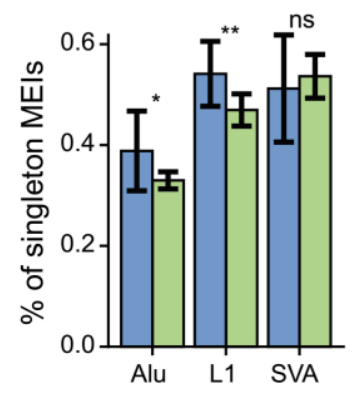

$\mathrm{E}$

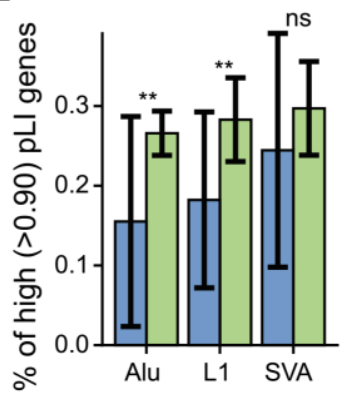

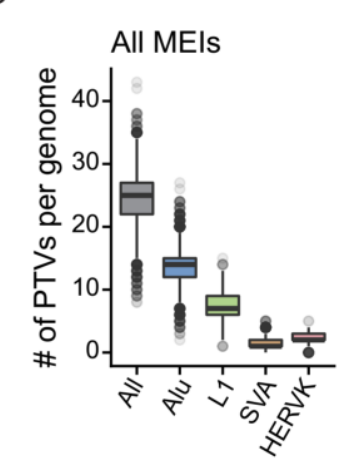

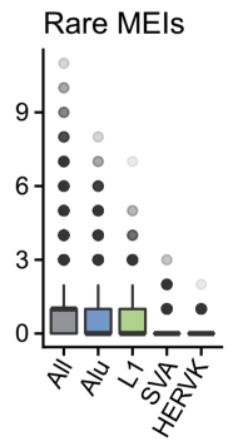

$\mathrm{F}$

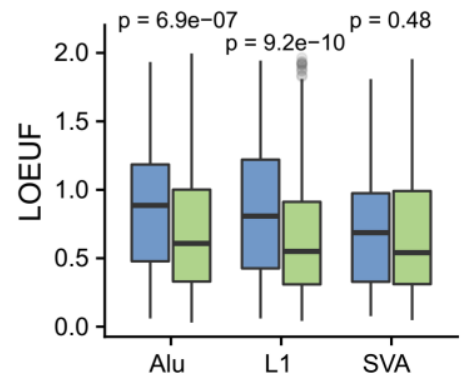

Coding Intron

Fig. 4. MEI functional properties. (A) Predicted functional consequences for each type of MEI: (left)

cumulative proportion, and (right) cumulative number. (B) Log2 fold enrichment of the MEI call set compared against the MEIs permutated. The permutation test was repeated 1000 times, and empirical p-

values were commutated together with the enrichment values. The enrichment values were scaled row- 
high pLI genes ( $\mathrm{pLI}>0.9)$ for genes with MEIs in the CDS and genes with MEIs intron regions. Error bars represent $95 \%$ CIs based on population proportion. P-values were computed using chi-squared test.

(F) Box plots of LOEUF scores of genes with MEIs in the CDS and genes with MEIs in their introns.

Wilcoxon rank sum test was used to compute p-values. Figure D-F used the same legend beneath. ns, $p$

$\geq 0.05 ; *, \mathrm{p}<0.05 ; * *, \mathrm{p}<0.01$

Although researchers have long noted that most of reference LTR elements and L1s in gene introns are in the antisense orientation with respect to the host genes (Smit 1999;

Medstrand et al. 2002), possibly due to ill effects on transcript processing of sense-oriented elements (van de Lagemaat et al. 2006; Zhang et al. 2011), there are no established conclusions about the orientation tendency of non-reference MEIs (Gardner et al. 2019; Hormozdiari et al. 2013). Our large collection of MEIs found in genes allowed us to closely examine the strand bias of different MEIs. Although a bias for Alu, L1 MEIs and SVA MEIs to be in an antisense orientation when found within genes was observed (Hormozdiari et al. 2013), we did not find a statistically significant bias for L1 insertions (Fig. S8A). Conversely, Alus were found to have strong strand bias when being inserted into protein-coding genes, non-coding genes, proteincoding introns, and non-coding introns (Fig. S8; $\chi 2 \mathrm{p}<0.05$ ). For SVA MEIs, protein-coding genes, protein-coding exons, and protein-coding introns were regions where insertion

261 orientation biases were detected (Fig. S8; $\chi^{2} \mathrm{p}<0.05$ ). Considering that Alu and SVA elements are non-autonomous TEs that are trans-mobilized by the L1 retrotransposition machinery 
which had MEIs in sense or antisense strand in introns did not show clear differences in terms

of selective constraints, by comparing the LOEUF scores of these two kinds genes (Fig. S8F).

In addition, no significant orientation tendency against the neighboring genes were detected when MEIs were in gene upstream regions (Fig. S8I). disease risk, suggesting their potential effects on common diseases (Payer and Burns 2019; Payer et al. 2017). To identify MEIs potentially associated with human trait or disease, we mapped MEIs to regions in linkage disequilibrium (LD) with trait- or disease-associated loci studies.

\section{L1 3' Transduction and 5' Inversion}

Some L1 elements can bring a 3' readthrough transcript to the offspring insert site, which is called 3' transduction (Goodier et al. 2000). These L1 elements are usually near a strong Poly(A) downstream L1 elements, we extracted the correspondence between L1s in different genomic 
Most of the sources transducts less than 20 offspring whereas site chrX:11713279 has 186

A

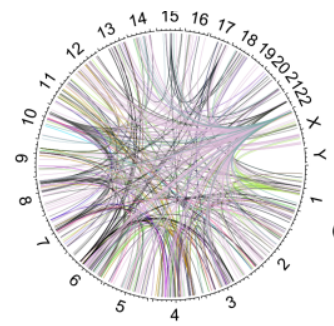

D

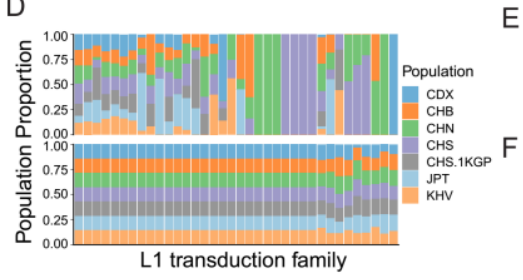

B

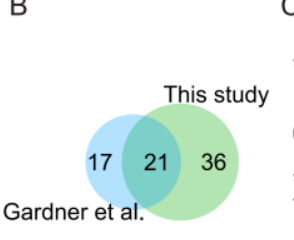

E

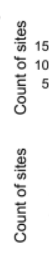

C

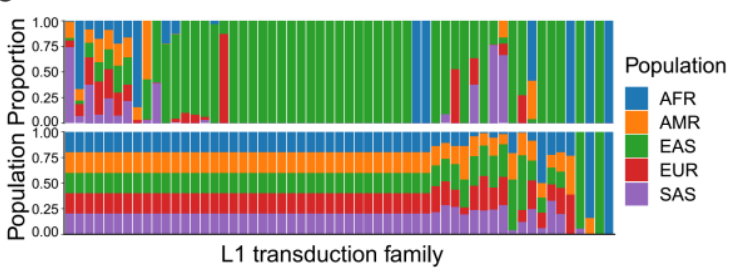

G

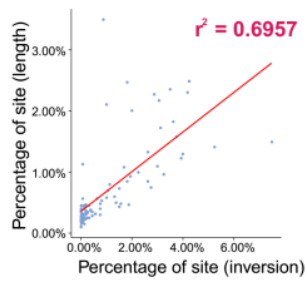

Fig. 5. L1 3' Transduction and 5' Inversion. (A) 3' transduction source-offspring relations across the 

indicate that the inversion process requires at least $\sim 600 \mathrm{bp}$ DNA sequence. In the previous

317 study, the distribution of the 5' inversion positions highly correlated with the distribution of L1

318 MEI lengths. MEIs in our study also showed this trend ( $\mathrm{R}^{2}=0.696$; Fig. 5E-G). We next

319 calculated the percentage of 5' end inverted MEIs within each 3' transduction offspring class. 


\section{A Database for Polymorphic MEIs}

Currently, resources for polymorphic TE findings in human genomes are in high demand

(Goerner-Potvin and Bourque 2018). There were two dedicated databases for polymorphic

human MEIs: dbRIP (Wang et al. 2006) and euL1db (Mir et al. 2015). However, the former had

A

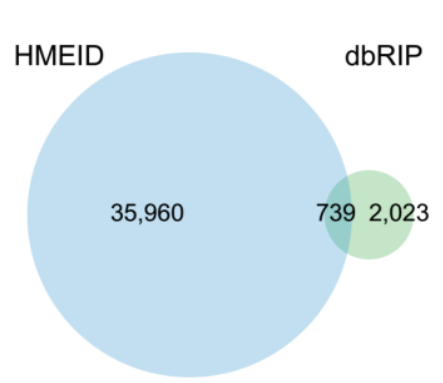

B HMEID (L1) euL1db

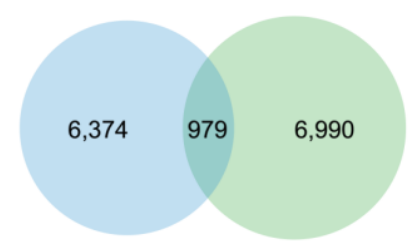

Fig. 6. Comparing HMEID with other MEI Databases. (A) Comparison the MEI set in the HMEID 


\section{Discussion}

341 MEIs, an endogenous and ongoing source of genetic variation, have not been investigated in

342 many population-scale WGS projects. Here we leveraged 5,675 genomes from the NyuWa

343 (Zhang et al. 2020) and the 1KGP (The 1000 Genomes Project Consortium 2015) dataset to

344 identify non-reference MEIs. After describing the frequency spectrum of variants, we focused on the insertion site preference and functional impacts of MEIs. We provided an important resource of non-reference MEIs in humans.

We identified 36,699 non-reference MEIs for four types of TEs and determined that individuals harbour a mean of over 1,000 non-reference MEIs, mostly contributed by Alu insertions. In line with previous reports (Gardner et al. 2017, 2019; Stewart et al. 2011), most MEIs were rare and individual-specific, which was also observed for SNVs (The 1000 of Chinese as well as East Asians. Comparing to the previous study conducted by the 1KGP (Gardner et al. 2017), the number of MEIs detected by us has increased about 55\%, representing what is to our knowledge the most comprehensive set of human non-reference MEIs.

We found that non-reference MEIs have non-random distributions along chromosomes, implicating the role of chromosome context in TE insertion. Of note, we found that nonreference L1 MEIs were drastically enriched in centromere regions, which was also supported 
1978), which could confer insertion preference for L1s, since the target specificity of L1 insertion machinery is TTTT/A (Feng et al. 1996). Certain centromeric histones and other centromeric proteins may also serve as preferred targets for TEs, as suggested by a study in maize (Schneider et al. 2016). Additionally, studies on HIV integration into the host genome implied that proximity to the nuclear periphery of centromere may facilitate TE targeting (Lelek et al. 2015; Marini et al. 2015). On the other hand, incorporation of L1s may facilitate the recurring evolutionary novelty of centromeres (Klein and O'Neill 2018). In support of this,

Evidences were also found in the tammar wallaby (Macropus eugenii), where dramatic enrichment of L1s and endogenous retroviruses was found in a latent centromere site (Longo et al. 2009), and Equus caballus, where evolutionarily new centromeres locate in LINE- and AT-rich regions (Nergadze et al. 2018). In addition to centromere ontogenesis, a LINE-like element (G2/jockey3) contributes directly to the organization and function of centromeres of D. melanogaster (Chang et al. 2019). This is also likely true for the non-reference SVA, for which we found an enrichment in telomeres, as TEs were found to be essential in maintaining the telomere length homeostasis in insects (Pardue and DeBaryshe 2011). However, another plausible explanation for both the enrichment of non-reference L1 MEIs in centromere and nonreference SVA MEIs in telomere is that these regions contain few protein-coding genes, limiting 
We have estimated that MEIs accounted for about $9.3 \%$ of all protein-truncating variants per polymorphic MEIs mapping to loci implicated in trait/disease association by GWAS, as

GWAS have mainly focused on small variants (Visscher et al. 2017), future association studies should consider and evaluate the effects of MEIs in common disease. We anticipate that the HMEID will serve as a basis for such studies. performance of MELT outperformed existing MEI discovery tools (Gardner et al. 2017) and it has been successfully used in several large-scale studies (Gardner et al. 2017, 2019; Feusier et al. 2019; Werling et al. 2018; Torene et al. 2020), but the detection power could be compromised by modest sequencing depth and incompetence in complex genomic regions of short-read WGS etc. In addition, the overall genotyping accuracy by MELT v2 was $87.95 \%$ for non-reference

401 Alus (not excluding MEIs in low complexity regions), when compared with PCR generated 402 genotypes (Goubert et al. 2020). As such, we have tried to ensure the site quality by strict 403 filtering. In the future, we would consider combining different MEI identification and genotyping tools to improve the quality, which has been proved useful in previous reports 
406

407

408

409

410

\section{Methods}

412 2,691

Also, long-read WGS is promising in detecting MEIs, especially for genomic regions refractory to approaches using short-read sequencing technologies (Audano et al. 2019; Chaisson et al. 2019; Zhou et al. 2020). Another limitation of our MEI dataset is that reference MEIs (MEIs detected as deletions) were not included yet, for which the detection is underway and the results would be integrated into the HMEID for public use.

\section{Experimental design}

Data in this study were from two sources: low-coverage ( 7.4X) WGS samples from the 1KGP (The 1000 Genomes Project Consortium 2015) and high-coverage ( 26.2X) WGS samples from the NyuWa dataset (Zhang et al. 2020). For the 1KGP dataset, CRAM-format files of individuals

were

downloaded

from

http://ftp.1000genomes.ebi.ac.uk/vol1/ftp/data_collections/1000_genomes_project/, which were aligned to human genome build GRCh38 (Lowy-Gallego et al. 2018). The CRAM files were then converted to BAMs using SAMtools v1.9 ( $\mathrm{Li}$ et al. 2009). The NyuWa dataset contained 2,999 individuals including diabetes and control samples collected from different provinces in China (Zhang et al. 2020), and this cohort was sequenced using the Illumina platform. The processing from raw FASTQs to BAMs was according to the GATK Best Practices Workflows germline short variant discovery pipeline (Poplin et al. 2018), as described in (Zhang et al. 2020). The median depth of the NyuWa samples after genome alignment (GRCh38 human genome build) and removal of PCR duplicates was about 26.2X. 


\section{Generation of MEI call set}

MELT v2.15 (Gardner et al. 2017) was run with default parameters using "SPLIT" mode to identify non-reference MEIs, which detects a wide range of non-reference $A l u$, L1, SVA and HERV-K insertions. To get the BAM coverage for MELT analysis, we used goleft v0.1.8 (https://github.com/brentp/goleft) "covstats" function to estimate the genomic coverage for each sample. After initial generation of a unified VCF file by MELT "MakeVCF" function, variants that did not pass the following criteria were filtered to get a high-quality MEI call set: 1) not in low complexity regions; 2) be genotyped in greater than $25.0 \%$ of individuals; 3 ) split reads > 2; 4) MELT ASSESS score > 3; and 5) VCF FILTER column be PASS. 2,998 of 2,999 samples in NyuWa and 2,677 of 2,691 samples in 1KGP were successfully analyzed, with the final call set consisting of 36,699 MEIs from 5,675 genomes. Subfamily characterization for Alu MEIs and L1 MEIs was done using MELT's CALU tool.

\section{Detection of L1 3' transduction and 5' inversion}

Following the generation of a high-quality MEI call set, MELT v2.15 was used to detect L1 3' transduction. We followed the instruction of MELT 3' transduction identification pipeline and extracted the METRANS and MESOURCE field in the resulting VCF manually. The population frequency was calculated with the AC/AN (for offspring MEI set, we used the sum of $\mathrm{AC}$ and $\mathrm{AN}$ ) and normalized across different populations.

The MELT VCF provided the position of a 5' inversion site (from the 3' end) through the "ISTP" field. We subtracted it from the full length of L1 (6,019 bp) to obtain the coordinate of the inversion site from the 5 ' end. While comparing the inversion coordinate and the length of 
L1, we removed the full-length L1 elements from the comparison set. Sites were distributed into 100 bins across the full length of L1. We compared the distribution of sequence length and inversion site position among these bins and calculated the Pearson correlation value.

\section{Analysis of Hardy-Weinberg equilibrium}

To evaluate the genotype distributions of each MEI under the null expectations set by the Hardy-Weinberg equilibrium (HWE), we tabulated genotype distributions of autosomal MEIs per dataset and performed exact tests by "HWExactStats" function in $\mathrm{R}$ package HardyWeinberg v1.6.3 (Graffelman 2015). While disequilibrium may indicate disease association or population stratification, it may be the result of confusion of heterozygotes and homozygotes. We thus used the HWE test for gross quality-check of genotyping accuracy (Fig. S2), as described in (Collins et al. 2020).

\section{Comparison with the 1 KGP MEI call set}

To compare with the MEIs generated by the 1KGP (Gardner et al. 2017), we downloaded the GRCh38 version call set from the dbVar database (Lappalainen et al. 2013). Then non-reference MEIs were extracted and compared with the MEIs identified in this study, using "window" function from BEDtools v2.26.0 (Quinlan and Hall 2010). When a site was located in $\pm 500 \mathrm{bp}$ of another site, it was considered as a hit.

\section{Testing MELT for different genome build and joint calling}

To test MELT's performance on different genome build, we randomly generated 100 samples from the 1KGP dataset, and we got the alignment files for both GRCh37 and GRCh38 version 
BEDtools v2.26.0 (Quinlan and Hall 2010).

To test MELT' performance with respect to sample size (joint calling), we randomly

\section{Functional annotation}

Variant Effect Predictor v99.2 (VEP) (McLaren et al. 2016) with Ensembl database version 99

(Zerbino et al. 2018) was used to annotate MEIs, with parameters “--pick --canonical --distance

a MEI was also found in non-coding genes and intergenic regions. 
the median length of all predicted LD regions as the block length, centered by the target SNP.

MEIs falling into these LD block regions. The complete set of these MEIs could be found in

Table S5.

To qualify the enrichment of MEIs across different genomic features (Fig. 4B), we

permuted 1,000 times for each MEI type with the same number as the real calls using GAT

v1.3.4 (Heger et al. 2013). Each permutation set was annotated with VEP and BEDtools using verify the results.

\section{Chromosome-level analyses of MEI density}

To check the distribution of MEIs throughout the genome, we used the method described by

Collins et al. (Collins et al. 2020) and we repeated it here for clarity. Focusing on 22 autosomes,

each chromosome was segmented into consecutive $100 \mathrm{~kb}$ bins and bins overlapped with 
Fig. 2, the normalized bin positions (i.e., -1 to 1 ) were cut into 500 uniform intervals, and values across all autosomes based on the normalized interval position were averaged. For the comparison of chromosome contexts (Fig. 2), normalized positions within the outermost $5 \%$ of

512 each chromosome arm were considered as "telomeric", the innermost $5 \%$ as "centromeric" and the other $90 \%$ of each arm as "interstitial". Visualization of density of different MEIs on each chromosome shown in Fig. S6 was done using RIdeogram v0.2.2 (Hao et al. 2020).

\section{Mutation rates}

Before estimating mutation rate, we exclude the MEIs failed in the HWE test (adjusted $\mathrm{p}<$ then used to estimate the genome mutation rate of each MEI type:

where $K$ is the number of MEI site observed per MEI type in given population, and is the total number of chromosomes assessed. Then mutation rates were estimated as:

$$
\widehat{\theta_{\mathrm{w}}}=4 N_{\mathrm{e}}
$$

524 with an effective population size (i.e. $N_{e}$ ) of 10,000, consistent with previous studies (Sudmant et al. 2015; Gardner et al. 2019; Collins et al. 2020). To estimate mutation rates worldwide, the 


\section{SNP heterozygosity and MEI diversity}

As described in a previous study (Hormozdiari et al. 2013), SNP heterozygosity was computed as the ratio of heterozygous SNPs over the length of the genome, and the mean value was used when multiple samples were considered. MEI diversity was defined as the average number of MEI differences between individuals in a population. For the NyuWa dataset (Zhang et al. 2020), high-quality SNP calls generated by the GATK v3.7 cohort pipeline (DePristo et al. 2011; Poplin et al. 2018) were used. For 1KGP3 samples, SNP calls on the human genome build GRCh38 of the were from http://ftp.1000genomes.ebi.ac.uk/vol1/ftp/data_collections/1000_genomes_project/release/20 190312 biallelic_SNV_and_INDEL/. Number of heterozygous SNPs was computed by VCFtools v0.1.15 (Danecek et al. 2011) and MEI diversity by "gtcheck" function in BCFtools v1.3.1 (Danecek and McCarthy 2017).

\section{Database construction}

We constructed the database with Bootstrap and Django. For each population, we calculated allele frequency of each MEI. All the data can be browsed in the database and downloaded from the "Download" page.

\section{Statistical analysis}

All statistical analyses in this study were briefly described in the main text and performed using $\mathrm{R}$ v3.6.2 (http://CRAN.R-project.org/). 


\section{Data Access}

548 Complete MEI call set and other related information such as allele frequency and functional annotation are available in the companion database HMEID (available at

\section{Acknowledgments}

We thank Eugene J. Gardner for helping us in using MELT. We thank Jing Wang for valuable comments in the data analysis and critical review of the manuscript. We thank Tingrui Song for assisting the use of high-performance computing platforms. We thank the people for generously contributing samples and sequencing data to the NyuWa dataset and the 1KGP dataset. Data analysis and computing resources were supported by the Center for Big Data Research in Health

557 (http://bigdata.ibp.ac.cn), Institute of Biophysics, Chinese Academy of Sciences. This work

\section{Author Contributions}

T.X. and S.M.H. conceptualized and supervised the project. Y.W.N., X.Y.T., Y.R.S., Y.Y.L., 
The authors declare no competing interests.

\section{References}

Audano PA, Sulovari A, Graves-Lindsay TA, Cantsilieris S, Sorensen M, Welch AE, Dougherty ML, Nelson BJ, Shah A, Dutcher SK, et al. 2019. Characterizing the Major Structural Variant Alleles of the Human Genome. Cell 0. https://www.cell.com/cell/abstract/S00928674(18)31633-7 (Accessed January 21, 2019).

Bennett EA, Keller H, Mills RE, Schmidt S, Moran JV, Weichenrieder O, Devine SE. 2008. Active Alu retrotransposons in the human genome. Genome Res 18: 1875-1883.

Buniello A, MacArthur JAL, Cerezo M, Harris LW, Hayhurst J, Malangone C, McMahon A, Morales J, Mountjoy E, Sollis E, et al. 2019. The NHGRI-EBI GWAS Catalog of published genome-wide association studies, targeted arrays and summary statistics 2019. Nucleic Acids Res 47: D1005-D1012.

Cao Y, Li L, Xu M, Feng Z, Sun X, Lu J, Xu Y, Du P, Wang T, Hu R, et al. 2020. The ChinaMAP analytics of deep whole genome sequences in 10,588 individuals. Cell Res 1-15.

Chaisson MJP, Sanders AD, Zhao X, Malhotra A, Porubsky D, Rausch T, Gardner EJ, Rodriguez OL, Guo L, Collins RL, et al. 2019. Multi-platform discovery of haplotype-resolved structural variation in human genomes. Nature Communications 10: 1784.

Chang CC, Chow CC, Tellier LC, Vattikuti S, Purcell SM, Lee JJ. 2015. Second-generation PLINK: rising to the challenge of larger and richer datasets. Gigascience 4. https://academic.oup.com/gigascience/article/4/1/s13742-015-0047-8/2707533 (Accessed June 29, 2019).

Chang C-H, Chavan A, Palladino J, Wei X, Martins NMC, Santinello B, Chen C-C, Erceg J, Beliveau BJ, Wu C-T, et al. 2019. Islands of retroelements are major components of Drosophila centromeres. PLOS Biology 17: e3000241.

Chueh AC, Northrop EL, Brettingham-Moore KH, Choo KHA, Wong LH. 2009. LINE Retrotransposon RNA Is an Essential Structural and Functional Epigenetic Component of a Core Neocentromeric Chromatin. PLOS Genetics 5: e1000354.

Collins RL, Brand H, Karczewski KJ, Zhao X, Alföldi J, Francioli LC, Khera AV, Lowther C, Gauthier LD, Wang H, et al. 2020. A structural variation reference for medical and population genetics. Nature 581: 444-451. 
F, Dube D, Chan SM, Robinson D, Meng F, et al. 2013. HIV infection reveals widespread expansion of novel centromeric human endogenous retroviruses. Genome Res 23: 15051513.

Cordaux R, Batzer MA. 2009. The impact of retrotransposons on human genome evolution. Nature Reviews Genetics 10: 691-703.

Danecek P, Auton A, Abecasis G, Albers CA, Banks E, DePristo MA, Handsaker RE, Lunter G, Marth GT, Sherry ST, et al. 2011. The variant call format and VCFtools. Bioinformatics 27: 2156-2158.

Danecek P, McCarthy SA. 2017. BCFtools/csq: haplotype-aware variant consequences. Bioinformatics 33: 2037-2039.

Deininger PL, Moran JV, Batzer MA, Kazazian HH. 2003. Mobile elements and mammalian genome evolution. Current Opinion in Genetics \& Development 13: 651-658.

DePristo MA, Banks E, Poplin R, Garimella KV, Maguire JR, Hartl C, Philippakis AA, del Angel G, Rivas MA, Hanna M, et al. 2011. A framework for variation discovery and genotyping using next-generation DNA sequencing data. Nat Genet 43: 491-498.

Dewannieux M, Esnault C, Heidmann T. 2003. LINE-mediated retrotransposition of marked Alu sequences. Nature Genetics 35: 41-48.

Ewing AD. 2015. Transposable element detection from whole genome sequence data. Mob DNA 6. http://www.ncbi.nlm.nih.gov/pmc/articles/PMC4696183/ (Accessed May 29, 2017).

Feng Q, Moran JV, Kazazian HH, Boeke JD. 1996. Human L1 Retrotransposon Encodes a Conserved Endonuclease Required for Retrotransposition. Cell 87: 905-916.

Feusier J, Watkins WS, Thomas J, Farrell A, Witherspoon DJ, Baird L, Ha H, Xing J, Jorde LB. 2019. Pedigree-based estimation of human mobile element retrotransposition rates. Genome Res 29: 1567-1577.

Fishilevich S, Nudel R, Rappaport N, Hadar R, Plaschkes I, Iny Stein T, Rosen N, Kohn A, Twik M, Safran M, et al. 2017. GeneHancer: genome-wide integration of enhancers and target genes in GeneCards. $\quad$ Database $\quad 2017$. https://academic.oup.com/database/article/doi/10.1093/database/bax028/3737828 (Accessed November 27, 2018).

Gardner EJ, Lam VK, Harris DN, Chuang NT, Scott EC, Pittard WS, Mills RE, Consortium 1000 Genomes Project, Devine SE. 2017. The Mobile Element Locator Tool (MELT): Population-scale mobile element discovery and biology. Genome Res gr.218032.116.

Gardner EJ, Prigmore E, Gallone G, Danecek P, Samocha KE, Handsaker J, Gerety SS, Ironfield H, Short PJ, Sifrim A, et al. 2019. Contribution of retrotransposition to developmental disorders. Nat Commun 10: 1-10. 
Goerner-Potvin P, Bourque G. 2018. Computational tools to unmask transposable elements. Nature Reviews Genetics 19: 688-704.

Goodier JL. 2016. Restricting retrotransposons: a review. Mobile DNA 7: 16.

Goodier JL, Ostertag EM, Kazazian Jr HH. 2000. Transduction of 3'-flanking sequences is common in L1 retrotransposition. Human Molecular Genetics 9: 653-657.

Goubert C, Thomas J, Payer LM, Kidd JM, Feusier J, Watkins WS, Burns KH, Jorde LB, Feschotte C. 2020. TypeTE: a tool to genotype mobile element insertions from whole genome resequencing data. Nucleic Acids Research. https://doi.org/10.1093/nar/gkaa074 (Accessed March 1, 2020).

Graffelman J. 2015. Exploring Diallelic Genetic Markers: The HardyWeinberg Package. Journal of Statistical Software 64: 1-23.

Hancks DC, Kazazian HH. 2016. Roles for retrotransposon insertions in human disease. Mob DNA 7. https://www.ncbi.nlm.nih.gov/pmc/articles/PMC4859970/ (Accessed June 20, 2019).

Hao Z, Lv D, Ge Y, Shi J, Weijers D, Yu G, Chen J. 2020. RIdeogram: drawing SVG graphics to visualize and map genome-wide data on the idiograms. PeerJ Comput Sci 6: e251.

Hedges DJ, Callinan PA, Cordaux R, Xing J, Barnes E, Batzer MA. 2004. Differential Alu Mobilization and Polymorphism Among the Human and Chimpanzee Lineages. Genome Res 14: 1068-1075.

Heger A, Webber C, Goodson M, Ponting CP, Lunter G. 2013. GAT: a simulation framework for testing the association of genomic intervals. Bioinformatics 29: 2046-2048.

Hormozdiari F, Konkel MK, Prado-Martinez J, Chiatante G, Herraez IH, Walker JA, Nelson B, Alkan C, Sudmant PH, Huddleston J, et al. 2013. Rates and patterns of great ape retrotransposition. PNAS 110: 13457-13462.

Huang CRL, Burns KH, Boeke JD. 2012. Active Transposition in Genomes. Annu Rev Genet 46: $651-675$.

Karczewski KJ, Francioli LC, Tiao G, Cummings BB, Alföldi J, Wang Q, Collins RL, Laricchia KM, Ganna A, Birnbaum DP, et al. 2020. The mutational constraint spectrum quantified from variation in 141,456 humans. Nature 581: 434-443.

Klein SJ, O’Neill RJ. 2018. Transposable elements: genome innovation, chromosome diversity, and centromere conflict. Chromosome Res 26: 5-23.

Kumar S, Subramanian S. 2002. Mutation rates in mammalian genomes. PNAS 99: 803-808.

Lander ES, Linton LM, Birren B, Nusbaum C, Zody MC, Baldwin J, Devon K, Dewar K, Doyle M, FitzHugh W, et al. 2001. Initial sequencing and analysis of the human genome. Nature 409: 860-921. 
Lappalainen I, Lopez J, Skipper L, Hefferon T, Spalding JD, Garner J, Chen C, Maguire M, Corbett M, Zhou G, et al. 2013. dbVar and DGVa: public archives for genomic structural variation. Nucleic Acids Res 41: D936-D941.

Lek M, Karczewski KJ, Minikel EV, Samocha KE, Banks E, Fennell T, O’Donnell-Luria AH, Ware JS, Hill AJ, Cummings BB, et al. 2016. Analysis of protein-coding genetic variation in 60,706 humans. Nature 536: 285-291.

Lelek M, Casartelli N, Pellin D, Rizzi E, Souque P, Severgnini M, Di Serio C, Fricke T, DiazGriffero F, Zimmer C, et al. 2015. Chromatin organization at the nuclear pore favours HIV replication. Nature Communications 6: 6483.

Li H. 2014. Toward better understanding of artifacts in variant calling from high-coverage samples. Bioinformatics 30: 2843-2851.

Li H, Handsaker B, Wysoker A, Fennell T, Ruan J, Homer N, Marth G, Abecasis G, Durbin R. 2009. The Sequence Alignment/Map format and SAMtools. Bioinformatics 25: 2078-2079.

Longo MS, Carone DM, Green ED, O’Neill MJ, O'Neill RJ, NISC Comparative Sequencing Program. 2009. Distinct retroelement classes define evolutionary breakpoints demarcating sites of evolutionary novelty. BMC Genomics 10: 334.

Lowy-Gallego E, Fairley S, Zheng-Bradley H, Clarke L, Flicek P. 2018. Variant calling on the GRCh38 assembly with the data from phase three of the 1000 Genomes. F1000Research 7. https://f1000research.com/posters/7-1445 (Accessed May 18, 2020).

Manuelidis L, Wu JC. 1978. Homology between human and simian repeated DNA. Nature 276: 92 94.

Marini B, Kertesz-Farkas A, Ali H, Lucic B, Lisek K, Manganaro L, Pongor S, Luzzati R, Recchia A, Mavilio F, et al. 2015. Nuclear architecture dictates HIV-1 integration site selection. Nature 521: 227-231.

McLaren W, Gil L, Hunt SE, Riat HS, Ritchie GRS, Thormann A, Flicek P, Cunningham F. 2016. The Ensembl Variant Effect Predictor. Genome Biology 17: 122.

Medstrand P, Lagemaat LN van de, Mager DL. 2002. Retroelement Distributions in the Human Genome: Variations Associated With Age and Proximity to Genes. Genome Res 12: 14831495.

Miga KH, Koren S, Rhie A, Vollger MR, Gershman A, Bzikadze A, Brooks S, Howe E, Porubsky D, Logsdon GA, et al. 2020. Telomere-to-telomere assembly of a complete human X chromosome. Nature 1-9.

Mills RE, Bennett EA, Iskow RC, Devine SE. 2007. Which transposable elements are active in the human genome? Trends in Genetics 23: 183-191. 
Mir AA, Philippe C, Cristofari G. 2015. euL1db: the European database of L1HS retrotransposon insertions in humans. Nucleic Acids Res 43: D43-D47.

Nergadze SG, Piras FM, Gamba R, Corbo M, Cerutti F, McCarter JGW, Cappelletti E, Gozzo F, Harman RM, Antczak DF, et al. 2018. Birth, evolution, and transmission of satellite-free mammalian centromeric domains. Genome Res 28: 789-799.

Ostertag EM, Kazazian HH. 2001. Twin Priming: A Proposed Mechanism for the Creation of Inversions in L1 Retrotransposition. Genome Res 11: 2059-2065.

Pardue M-L, DeBaryshe PG. 2011. Retrotransposons that maintain chromosome ends. PNAS 108: 20317-20324.

Payer LM, Burns KH. 2019. Transposable elements in human genetic disease. Nat Rev Genet 20: 760-772.

Payer LM, Steranka JP, Yang WR, Kryatova M, Medabalimi S, Ardeljan D, Liu C, Boeke JD, Avramopoulos D, Burns KH. 2017. Structural variants caused by Alu insertions are associated with risks for many human diseases. PNAS 114: E3984-E3992.

Poplin R, Ruano-Rubio V, DePristo MA, Fennell TJ, Carneiro MO, Auwera GAV der, Kling DE, Gauthier LD, Levy-Moonshine A, Roazen D, et al. 2018. Scaling accurate genetic variant discovery to tens of thousands of samples. bioRxiv 201178.

Prado-Martinez J, Sudmant PH, Kidd JM, Li H, Kelley JL, Lorente-Galdos B, Veeramah KR, Woerner AE, O'Connor TD, Santpere G, et al. 2013. Great ape genetic diversity and population history. Nature 499: 471-475.

Quinlan AR, Hall IM. 2010. BEDTools: a flexible suite of utilities for comparing genomic features. Bioinformatics 26: 841-842.

Raiz J, Damert A, Chira S, Held U, Klawitter S, Hamdorf M, Löwer J, Strätling WH, Löwer R, Schumann GG. 2012. The non-autonomous retrotransposon SVA is trans -mobilized by the human LINE-1 protein machinery. Nucleic Acids Res 40: 1666-1683.

Rishishwar L, Mariño-Ramírez L, Jordan IK. 2016. Benchmarking computational tools for polymorphic transposable element detection. Brief Bioinform. https://academic.oup.com/bib/article/doi/10.1093/bib/bbw072/2562836 (Accessed October 31, 2017).

Schneider KL, Xie Z, Wolfgruber TK, Presting GG. 2016. Inbreeding drives maize centromere evolution. PNAS 113: E987-E996.

Smit AF. 1999. Interspersed repeats and other mementos of transposable elements in mammalian genomes. Current Opinion in Genetics \& Development 9: 657-663. 
HYK, Lee W-P, et al. 2011. A Comprehensive Map of Mobile Element Insertion $\begin{array}{lllll}\text { Polymorphisms in Humans. PLoS } & \text { Genet }\end{array}$ https://www.ncbi.nlm.nih.gov/pmc/articles/PMC3158055/ (Accessed March 10, 2020).

Sudmant PH, Rausch T, Gardner EJ, Handsaker RE, Abyzov A, Huddleston J, Zhang Y, Ye K, Jun G, Hsi-Yang Fritz M, et al. 2015. An integrated map of structural variation in 2,504 human genomes. Nature 526: 75-81.

Sultana T, Zamborlini A, Cristofari G, Lesage P. 2017. Integration site selection by retroviruses and transposable elements in eukaryotes. Nature Reviews Genetics 18: 292-308.

The 1000 Genomes Project Consortium. 2015. A global reference for human genetic variation. Nature 526: 68-74.

Torene RI, Galens K, Liu S, Arvai K, Borroto C, Scuffins J, Zhang Z, Friedman B, Sroka H, Heeley J, et al. 2020. Mobile element insertion detection in 89,874 clinical exomes. Genet Med 15 .

van de Lagemaat LN, Medstrand P, Mager DL. 2006. Multiple effects govern endogenous retrovirus survival patterns in human gene introns. Genome Biol 7: R86.

Visscher PM, Wray NR, Zhang Q, Sklar P, McCarthy MI, Brown MA, Yang J. 2017. 10 Years of GWAS Discovery: Biology, Function, and Translation. The American Journal of Human Genetics 101: 5-22.

Wang J, Song L, Grover D, Azrak S, Batzer MA, Liang P. 2006. dbRIP: A Highly Integrated Database of Retrotransposon Insertion Polymorphisms in Humans. Hum Mutat 27: 323329.

Wang L, Norris ET, Jordan IK. 2017. Human Retrotransposon Insertion Polymorphisms Are Associated with Health and Disease via Gene Regulatory Phenotypes. Front Microbiol 8. https://www.frontiersin.org/articles/10.3389/fmicb.2017.01418/full (Accessed August 20, 2020).

Waterson RH, Lander ES, Wilson RK, The Chimpanzee Sequencing and Analysis Consortium. 2005. Initial sequence of the chimpanzee genome and comparison with the human genome. Nature 437: 69-87.

Watterson GA. 1975. On the number of segregating sites in genetical models without recombination. Theoretical Population Biology 7: 256-276.

Werling DM, Brand H, An J-Y, Stone MR, Zhu L, Glessner JT, Collins RL, Dong S, Layer RM, Markenscoff-Papadimitriou E, et al. 2018. An analytical framework for whole-genome sequence association studies and its implications for autism spectrum disorder. Nat Genet 50: $727-736$.

Wu D, Dou J, Chai X, Bellis C, Wilm A, Shih CC, Soon WWJ, Bertin N, Lin CB, Khor CC, et al. 
2019. Large-Scale Whole-Genome Sequencing of Three Diverse Asian Populations in Singapore. Cell 179: 736-749.e15.

Zahn J, Kaplan MH, Fischer S, Dai M, Meng F, Saha AK, Cervantes P, Chan SM, Dube D, Omenn GS, et al. 2015. Expansion of a novel endogenous retrovirus throughout the pericentromeres of modern humans. Genome Biology 16: 74.

Zerbino DR, Achuthan P, Akanni W, Amode MR, Barrell D, Bhai J, Billis K, Cummins C, Gall A, Girón CG, et al. 2018. Ensembl 2018. Nucleic Acids Res 46: D754-D761.

Zhang P, Luo H, Li Y, Wang Y, Wang J, Zheng Y, Niu Y, Shi Y, Zhou H, Song T, et al. 2020. NyuWa Genome Resource: Deep Whole Genome Sequencing Based Chinese Population Variation Profile and Reference Panel. bioRxiv 2020.11.10.376574.

Zhang Y, Romanish MT, Mager DL. 2011. Distributions of Transposable Elements Reveal Hazardous Zones in Mammalian Introns. PLOS Computational Biology 7: e1002046.

Zhou W, Emery SB, Flasch DA, Wang Y, Kwan KY, Kidd JM, Moran JV, Mills RE. 2020. Identification and characterization of occult human-specific LINE-1 insertions using longread sequencing technology. Nucleic Acids Res 48: 1146-1163.

2019. The GenomeAsia 100K Project enables genetic discoveries across Asia. Nature 576: 106-111. 